\title{
Apical aneurism and myocardial bridging in a patient with hypertrophic cardiomyopathy: Association or consequence of the myocardial bridging?
}

\author{
Anthony Foucault ${ }^{1}$, Loic Hilpert ${ }^{1}$, Francois Hédoire ${ }^{2}$, Eric Saloux ${ }^{1}$, Sophie Gomes ${ }^{1}$, \\ Arnaud Pellissier ${ }^{1}$, Patrice Scanu ${ }^{1}$, Laure Champ-Rigot ${ }^{1}$, Paul Milliez ${ }^{1}$ \\ ${ }^{1}$ Cardiology Department, Caen University Hospital, Normandy, France \\ ${ }^{2}$ Cardiology Department, Saint Martin Clinic, Caen, Normandy, France
}

\begin{abstract}
The identification of high-risk patients with hypertrophic cardiomyopathy (HC) for primary prevention of sudden cardiac death (SCD) remains a challenging issue, since major risk factors sometimes lack specificity. We report the case of a patient with HC and association of apical aneurysm and myocardial bridging who had been initially not implanted because she had only one major risk factor. She subsequently experienced a sustained ventricular tachycardia that finally motivated the implantation. We conclude that it is never an easy decision to implant a preventive implantable cardioverter-defibrillator (ICD). Nevertheless, additional criteria for a better selection of patients who would benefit from an ICD implant are certainly useful. (Cardiol J 2012; 19, 6: 643-645)
\end{abstract}

Key words: hypertrophic cardiomyopathy, sudden cardiac death, myocardial bridging

\section{Introduction}

Sudden cardiac death (SCD) is the most devastating and unpredictable outcome in patients suffering from hypertrophic cardiomyopathy (HC). Whereas in secondary prevention there is a consensus for an implantable cardioverter-defibrillator (ICD) [1], identification of high-risk patients for primary prevention of SCD remains a challenging issue. In clinical practice, patients may have only one of the recognized risk factors of SCD, and it is often difficult to largely implant an ICD in all these patients. Recent reports have focused on new risk markers, such as the presence of a myocardial bridging [2], a myocardial fibrosis [3] or an apical aneurysm [4]. We report here the case of a patient with $\mathrm{HC}$ and association of an apical aneurysm and myocardial bridging who had been initially not implanted, and who subsequently experienced a sustained ventricular tachycardia (VT) with a very high level of troponin that finally motivated the implantation.

\section{Case report}

The patient is a 48 year-old woman, who has been followed since 2001 for a HC. She had no personal history of syncope, no familial history of SCD or even HC. An initial echocardiography assessed a septo-apical dominant $\mathrm{HC}$ (thickness $17 \mathrm{~mm}$ ),

Address for correspondence: Anthony Foucault, MD, Cardiology Department, Caen University Hospital, Normandy, Avenue de la Cote de Nacre, 14033 Caen, France, tel: +332 310651 18, fax: +33231 0644 18,

e-mail: foucault-a@chu-caen.fr

Received: 11.07.2011 Accepted: 12.08.2011 


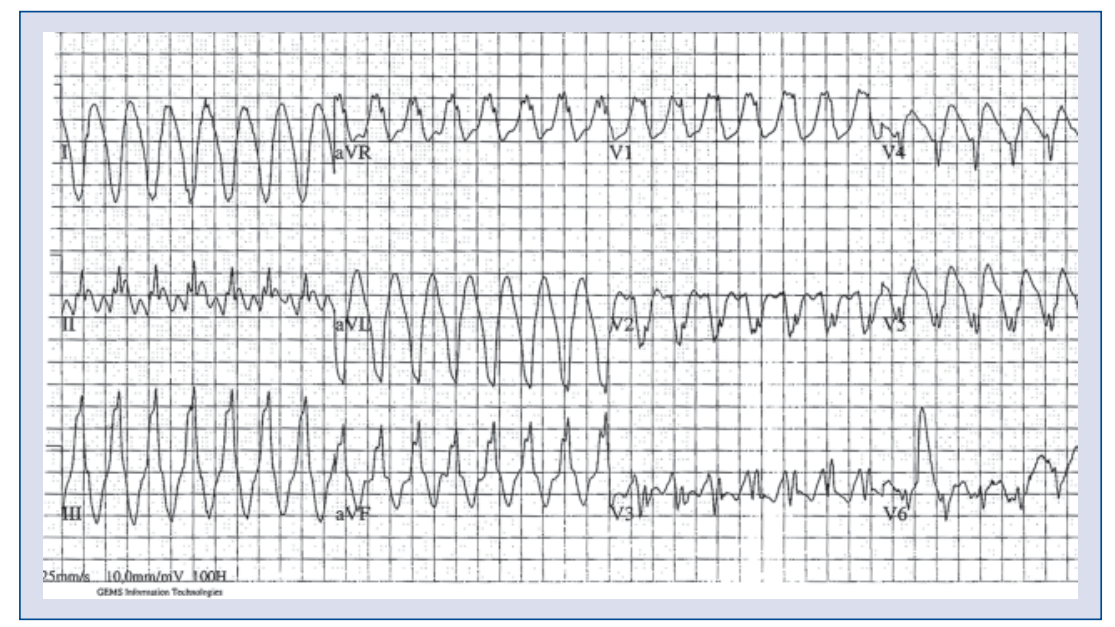

Figure 1. ECG when patient presented dizziness and palpitations with ventricular tachycardia.

posterior wall thickness of $16 \mathrm{~mm}$, and a left ventricular ejection fraction of $62 \%$. There was neither mitral valve systolic anterior motion, nor left ventricular outflow tract obstruction. At that time, she had non-sustained VT on a 24-hour ambulatory ECG recording, before any beta-blocker treatment was initiated.

Two years later, she consulted due to a transient diplopia: cerebral magnetic resonance imaging (MRI) was normal, but the echocardiography showed an increased hypertrophy with a $19 \mathrm{~mm}$ septum thickness and the appearance of an apical aneurysm. Cardiac MRI confirmed such an apical aneurysm, with a small thrombus and an apical delayed enhancement that could correspond to fibrosis or a healed myocardial infarction. As the patient was also an active smoker, a coronarography was performed and showed a mid left anterior descending (LAD) coronary artery bridging, but no coronary stenosis. We decided to begin anticoagulant medication.

Since this episode, the patient remained asymptomatic with normal treadmill test, repetitive Holter still showing mild ventricular excitability, and stable hypertrophy and fibrosis with no outflow obstruction on either contrast-enhanced echocardiography or the last cardiac MRI performed in July 2009. However, at that time she was referred in our institution to discuss a primary prevention of an ICD. Despite the association of one recognized risk factor of SCD (non-sustained VT) with this apical aneurysm, we postponed ICD implantation in this asymptomatic patient because of the unusual aspect of this aneurysm that differed from the description by Maron et al. [4].
In December 2010, the patient presented dizziness with palpitations. On physical examination, pulse was regular at $180 \mathrm{bpm}$ and blood pressure stable. Twelve-lead ECG revealed a monomorphic VT coming from the apical aspect of the left ventricle (Fig. 1). After restoration of sinus rhythm, we unexpectedly found a highly increased troponin level of $90 \mu \mathrm{g} / \mathrm{L}$. Coronarography still showed the known mid-LAD coronary artery bridging, without any coronary stenosis (Fig. 2). CMR showed a larger apical aneurysm with a transmural fibrosis and severe mid-ventricular hypertrophy with a septal thickness now at $26 \mathrm{~mm}$ (Fig. 2). We postulated that VT might be related to the natural history of this $\mathrm{HC}$ with an apical aneurysm, or because of this high level of troponin and this increasing apical aneurysm, secondary to an ischemic event due to this LAD bridging. After careful discussion, because of this large transmural scar with no evidence of viability at the MRI, neither LAD angioplasty nor surgical bypass was done, while the implantation of an ICD as secondary prevention was scheduled.

\section{Discussion}

The decision for preventive implantation of ICD in patients with $\mathrm{HC}$ is often difficult because patients frequently display only one recognized risk factor. Although a large cohort study has shown that one risk factor could justify ICD implantation [5], it is also important to keep in mind that the rate of complications related to the device is unacceptably high. And patients with HC may also suddenly die even in the absence of any risk marker [1]. As a result, it is of great importance to seek additional 


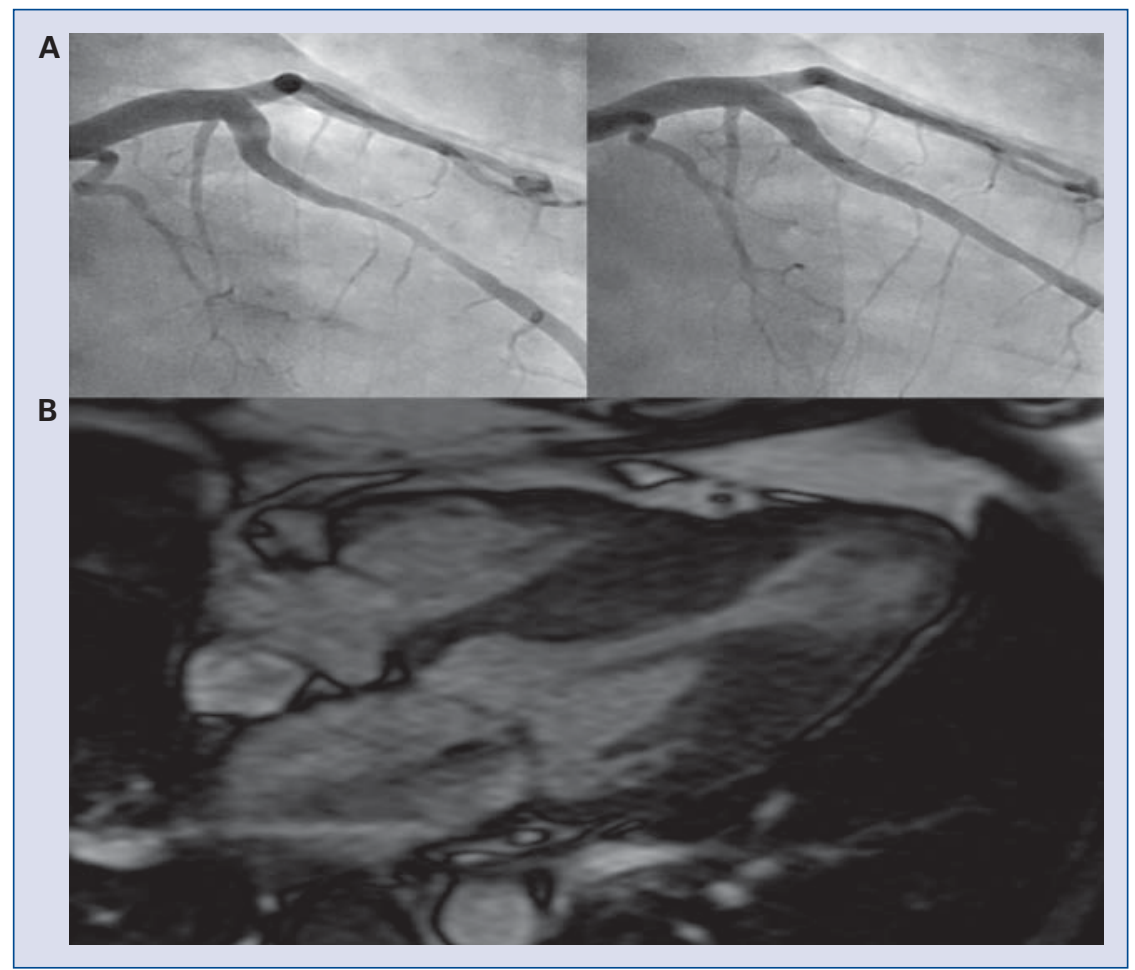

Figure 2. A. Coronarography showing myocardial bridging of the left anterior descending coronary artery in systole (left) and diastole (right); B. Cardiac resonance imaging showing predominant septal hypertrophy and apical aneurysm.

criteria for a better selection of patients who would benefit from an ICD implantation. Recently, the presence of a left ventricular apical aneurysm in $\mathrm{HC}$, a large zone of fibrosis or a myocardial bridging have been correlated to a higher SCD rate [2-4]. In this case report, the patient had both an apical aneurysm and a myocardial bridging.

Because of the late appearance of this aneurysm with initially a thrombus concomitantly to the occurrence of a stroke, and the recent VT episode associated with this high level of troponin, we thought that this apical aneurysm could be related to this LAD bridging and so is a consequence of repeated ischemic events.

Conflict of interest: none declared

\section{References}

1. Elliott PM, Poloniecki J, Dickie S et al. Sudden death in hypertrophic cardiomyopathy: Identification of high risk patients. J Am Coll Cardiol, 2000; 36: 2212-2218.

2. Basso C, Thiene G, Mackey-Bojack S, Frigo AC, Corrado D, Maron BJ. Myocardial bridging, a frequent component of the hypertrophic cardiomyopathy phenotype, lacks systematic association with sudden cardiac death. Eur Heart J, 2009; 30: 1627-1634.

3. O'Hanlon R, Grasso A, Roughton M et al. Prognostic significance of myocardial fibrosis in hypertrophic cardiomyopathy. J Am Coll Cardiol, 2010; 56: 867-874.

4. Maron MS, Finley JJ, Bos JM et al. Prevalence, clinical significance, and natural history of left ventricular apical aneurysms in hypertrophic cardiomyopathy. Circulation, 2008; 118: 1541-1549.

5. Maron BJ, Spirito P, Shen WK et al. Implantable cardioverter-defibrillators and prevention of sudden cardiac death in hypertrophic cardiomyopathy. JAMA, 2007; 298: 405-412. 